\title{
LOCALLY SOLUBLE GROUPS WITH ALL NONTRIVIAL NORMAL SUBGROUPS ISOMORPHIC
}

\author{
John C. Lennox, Howard Smith and James Wiegold
}

Abstract

Let $G$ be an infinite, locally soluble group which is isomorphic to all its nontrivial normal subgroups. If $G / G^{\prime}$ has finite $p$-rank for $p=0$ and for all primes $p$, then $G$ is cyclic.

In paper [2] we considered groups which are isomorphic to all of their nontrivial normal subgroups. The question as to which infinite groups have this property $P$, say, was first raised by Philip Hall. It was shown in [2] that, if $G$ is a finitely generated infinite $P$-group which contains a proper normal subgroup of finite index, then $G$ is cyclic, and our conjecture is that $\mathbb{Z}$ is the only finitely generated infinite $P$-group which is not simple. It was further remarked in [2] that is should perhaps be possible to deal with the locally soluble case, and this note represents a step in this direction. The following is proved.

Theorem. Let $G$ be an infinite, locally soluble group which is isomorphic to all of its nontrivial normal subgroups. If $G / G^{\prime}$ has finite $p$-rank for all $p=0$ or a prime then $G$ is cyclic.

We recall that an abelian group $A$ has $p$-rank $r$ if the cardinality of a maximal independent subset of elements of $A$ of order $p$ is equal to $r$. In particular, if $A$ has finite (Prüfer) rank then the $p$-ranks of $A$ are boundedly finite.

There is one aspect of the proof of our theorem which recalls part of the proof from [2], namely the exploitation of "linearity conditions" which are forced by the rank restrictions (in conjunction with property $P$ ). In the case where $G$ has normal abelian $p$-sections of possibly infinite rank, such a technique is bound to fail, and it is not clear how one might approach the case where, for example, $G$ is an arbitrary locally nilpotent 
group with $P$. Clearly such a group is either torsionfree or a $p$-group, but beyond that there is little that we can say at the moment.

\section{Proof of the theorem:}

Suppose first that $G$ has a nontrivial, torsionfree soluble image $S$ and let $r$ be the 0-rank of $G / G^{\prime}$. Then, because of property $P, S$ has finite Hirsch length (that is, the sum of the 0-ranks of the derived factors of $G$ is finite). Let $F$ denote the Fitting subgroup of $S$. Then $F$ is locally nilpotent and its abelian subgroups have finite 0-rank. Since $F$ is torsionfree, it is nilpotent (see Lemma 6.37 of [3]). Let $A$ be a maximal normal abelian subgroup of $F$. Then $A$ is self-centralizing in $F$ and of rank at most $r$ (again by $P$ ), and so $F / A$ embeds in the group of (upper) unitriangular $r \times r$ matrices over $\mathbb{Q}$. It follows that $F / A$ and hence $F$ has bounded rank and bounded nilpotency class $c$, say. For each $i=1, \ldots, c$, let $Z_{i}$ denote the $i$-th term of the upper central series of $F$ and let $D_{i}$ be the centralizer in $S$ of $Z_{i} / Z_{i-1}$ (where $Z_{0}=1$ ). Then $S / D_{i}$ is a soluble group of automorphisms of $Z_{i} / Z_{i-1}$, which is torsionfree abelian of rank at most $r$, and so $S / D_{i}$ embeds in $G L(r, \mathbb{Q})$. By the result of Zassenhaus ([3, Theorem 3.23]), $S / D_{i}$ has bounded derived length. Let $D=\bigcap_{i=1}^{c} D_{i}$. Then $S / D$ has bounded derived length. Further, $D$ stabilizes a series of length $c$ in $F$ and so, writing $C$ for the centralizer of $F$ in $S$, we see that $D / C$ is nilpotent ([1, Lemma 3.5]). But $C=Z(F)$ (e.g. Lemma 2.17 of $[3])$ and so $[C, D]=1$ and $D$ is nilpotent and hence in $F$. It follows that $S$ has bounded derived length and we can choose $N$ minimal subject to $N \triangleleft G$ and $G / N$ torsionfree soluble. If $N \neq 1$ then, by property $P, N$ has a nontrivial, torsionfree soluble image, contradicting the definition of $N$. Thus $N=1$ and $G$ is soluble. Clearly $G \cong \mathbb{Z}$ in this case. From now on, we may assume that all soluble images of $G$ are periodic. (If $G$ were to have a nonperiodic soluble image then some abelian normal factor of $G$ would be nontrivial and torsionfree and so, again by $P, G / G^{\prime}$ would have a nontrivial torsionfree image.) Let $H / K$ be an arbitrary chief factor of $G$ - such exists in every nontrivial group. Then $H / K$ is an elementary abelian $p$-group, for some prime $p$, and we see that $G$ therefore has a nontrivial finite $p$-image. Let $P_{1}=G^{\prime} G^{p}$ and, for $i \geq 1$, let $P_{i+1}=P_{i}^{\prime} P_{i}^{p}$. By property $P$, the subgroups $P_{i}$ form a strictly descending chain of normal subgroups of $G$. Also, each $G / P_{i}$ is a finite $p$-group. Let $R=\bigcap_{i=1}^{\infty} P_{i}$ and write $\bar{G}=G / R, \bar{P}_{i}=P_{i} / R, i=1,2, \ldots$ Let $s$ be the rank of $G / P_{1}$ and let $\bar{A}$ be an arbitrary finitely generated abelian subgroup of $\bar{G}$. The subgroups $\bar{A} \cap \bar{P}_{i}$ form a descending chain, with trivial intersection, such that each $\bar{A} / \bar{A} \cap \bar{P}_{i}$ is a finite (abelian) $p$-group of rank at most $s$ (since $\bar{A} \bar{P}_{i} / \bar{P}_{i}$ is subnormal in $\bar{G} / \bar{P}_{i}$ ). It 
follows that $\bar{A}$ has rank at most $s$, and so $\bar{G}$ is a locally soluble group whose abelian subgroups have bounded rank. By a result of Merzljakov (see p. 89 , vol. 2 of [3] for a reference), $\bar{G}$ has finite rank.

Now by Lemma 10.39 of [3], $\bar{G}$ is periodic-by-soluble and hence periodic. Clearly, therefore, $\bar{G}$ is a locally nilpotent $p$-group and hence a Černikov group (Corollary 1 to Theorem 6.36 of $[3]$ ). Since $\bar{G}$ is residually finite, it must be finite, contradicting the choice of the subgroups $P_{i}$. This completes the proof of the theorem.

\section{References}

1. PhiliP Hall, "The Edmonton notes on nilpotent groups," Queen Mary College Mathematics Notes, London, 1969.

2. John C. Lennox, Howard Smith And James Wiegold, A problem about normal subgroups, Journal of Pure and Applied Algebra 88 (1993), 169-171.

3. D. J. S. RoBinson, "Finiteness conditions and generalized soluble groups," Springer-Verlag, Berlin-Heidelberg-New York, 1972.

John C. Lennox:

School of Mathematics

University of Wales

College of Cardiff

Cardiff CF2 4AG

WALES
Howard Smith:

Department of Mathematics

Bucknell University

Lewisburg PA 17837

U.S.A.

James Wiegold:

School of Mathematics

University of Wales

College of Cardiff

Cardiff CF2 4AG

WALES

Rebut el 14 d'Octubre de 1993 